\title{
Synergistic effect of chidamide and venetoclax on apoptosis in acute myeloid leukemia cells and its mechanism
}

\author{
Gangping Li, Dongbei Li, Fangfang Yuan, Cheng Cheng, Lin Chen, Xudong Wei \\ Department of Hematopathy, Henan Institute of Hematology, The Affiliated Cancer Hospital of Zhengzhou University, Zhengzhou, China \\ Contributions: (I) Conception and design: X Wei; (II) Administrative support: D Li; (III) Provision of study materials or patients: C Cheng, L Chen; \\ (IV) Collection and assembly of data: F Yuan; (V) Data analysis and interpretation: G Li; (VI) Manuscript writing: All authors; (VII) Final approval of \\ manuscript: All authors. \\ Correspondence to: Xudong Wei. Department of Hematopathy, Henan Institute of Hematology, The Affiliated Cancer Hospital of Zhengzhou \\ University, Zhengzhou 450008, China. Email: xudongwei@zzu.edu.cn.
}

Background: Acute myeloid leukemia (AML) is a hematological malignancy with a low remission rate and
high recurrence rate. Overexpression of the antiapoptotic protein Bcl-2 is associated with a lower overall
survival rate in AML patients. Venetoclax (ABT199) is a selective inhibitor of Bcl-2 that has a significant
effect in AML, but single-drug resistance often occurs due to the high expression of Mcl-1 protein. Studies
have confirmed that chidamide can downregulate the expression levels of Bcl-2 and Mcl-1 and induce
apoptosis. Methods: This study aimed to use AML cell lines and primary cells to study the effects of venetoclax and chidamide combination therapy on AML cell apoptosis, the cell cycle, and changes in related signaling pathways in vitro; establish an AML mouse model to observe the efficacy and survival time of combination therapy in vivo; and analyze the drug effects with multi-omics sequencing technology. The changes in gene and protein expression before and after treatment were examined to clarify the molecular mechanism driving the synergistic effect of the two drugs.

Results: (I) Both venetoclax and chidamide promoted apoptosis in AML cell lines and primary cells in a time- and concentration-dependent manner. The effect was further enhanced when the two drugs were combined, and a synergistic effect was observed (combination index <1). (II) At both the mRNA and protein levels, the expression of Mcl-1 was upregulated by venetoclax and downregulated by chidamide, and the expression of Mcl-1 decreased further after combination treatment. (III) Transcriptome sequencing showed that differentially expressed genes in the combination group compared with the venetoclax monotherapy group were mainly enriched in the PI3K-AKT pathway and JAK2/STAT3 pathway. Moreover, qRT-PCR and Western blot confirmed these results. (IV) The combination therapy group exhibited significantly inhibited disease progression and a prolonged survival time among AML mice.

Conclusions: Chidamide combined with venetoclax synergistically promoted apoptosis in AML cell lines and primary cells by inhibiting activation of the PI3K/AKT pathway and JAK2/STAT3 pathway.

Keywords: Chidamide; venetoclax; acute myeloid leukemia (AML); apoptosis; mechanism

Submitted Sep 02, 2021. Accepted for publication Oct 16, 2021.

doi: 10.21037/atm-21-5066

View this article at: https://dx.doi.org/10.21037/atm-21-5066

\section{Introduction}

Acute myeloid leukemia (AML) is a genetically heterogeneous clonal malignancy originating from clonal hematopoietic stem cells and is characterized by chromosomal abnormalities, recurrent gene mutations, epigenetic modifications affecting chromatin structure, and microRNA dysregulation. Although the complete remission (CR) rate of AML has greatly improved with the emergence 
of new drugs, targeted drugs, and immunotherapy, $20-40 \%$ of patients still have difficulty achieving CR, and approximately $60 \%$ of patients eventually relapse (1-3). The 5-year overall survival rate of AML is only approximately $25 \%$ (3). Pre-clinical and clinical studies have demonstrated that the selective and highly potent Bcl-2 inhibitor venetoclax has anti-leukemia activities against various hematological malignancies, including myelodysplastic syndrome (MDS), chronic lymphocytic leukemia (CLL), and AML (4-8). Venetoclax is FDA approved for a subset of patients with CLL and AML $(9,10)$, and the FDA-approved combination of venetoclax with decitabine or azacitidine is resulting in CR/complete response with incomplete hematologic recovery (CRi) rates of $70-95 \%$ and good tolerability in elderly AML patients, although patients invariably relapse $(8,11,12)$. Resistance to apoptosis inducted by venetoclax in AML is mediated by pre-existing and venetoclax-induced overexpression of Mcl1 (8), and the overexpression of Mcl-1 has been reported in AML at relapse (13). The mechanism by which Mcl1 blocks the progression of apoptosis is through binding and sequestering the pro-apoptotic $\mathrm{BH} 3$-only proteins Bim, PUMA, Noxa, Bak, and Bax (14), preventing pore formation on the mitochondrial membrane and the release of cytochrome $\mathrm{c}$ into the cytoplasm (15). Therefore, it is important to identify clinically available agents that interfere with Mcl-1 to augment the therapeutic efficacy of venetoclax in AML (16), and numerous studies combining it with other therapies are being trialed $(12,17,18)$.

Cancer can be caused not only by changes in DNA sequences but by typical epigenetic modifications, such as histone modifications. This type of epigenetic modification can remodel chromatin, change the cell phenotype, regulate gene expression, and promote cancer development $(19,20)$. As acetylation modifications at specific sites can regulate the pathways involved in the cell cycle and apoptosis (21), the leukemia epigenome has become a new target for histone deacetylase inhibitors (HDACis) (22). Chidamide (CS055) is a HDACi that was independently developed in China and has been approved for the treatment of peripheral T cell lymphoma (PTCL) (23-26). Study has shown that chidamide can inhibit the proliferation of AML cells, at the same time block the cell cycle in G0/G1 phase, and induce cell apoptosis (27). Wang et al. found chidamide can improve the sensitivity of anthracyclines by inhibiting HDAC3-AKT-P21-CDK2 signal pathway in AML (28). Chidamide can enhance the cytotoxicity of cytarabine and sorafenib in AML cells (29). To further examine the synergistic killing effect and mechanism of combination therapy on AML, this study aimed to use AML cell lines and primary cells to study the effects of venetoclax and chidamide combination therapy on AML cell apoptosis, cell cycle, and changes in related signaling pathways in vitro; establish an AML mouse model to examine the efficacy and survival time of combination therapy in vivo; and analyze the drug effects with multi-omics sequencing technology. Transcriptome sequencing technology allows researchers to quickly obtain differential expression information of mRNA, and through functional analysis of differentially expressed genes, discover the characteristics of gene expression regulation changes in biological processes such as signal transduction. The changes in gene and protein expression before and after treatment were examined to clarify the molecular mechanism driving the synergistic effect of the two drugs to provide a new treatment strategy for clinical refractory/relapsed AML. In our study, we found that chidamide and venetoclax synergistically decrease the mitochondrial membrane potential. Most importantly, we focused on the mechanism of chidamide and venetoclax acting on AML, it was found by transcriptome sequencing and verified by Western blot and quantitative reverse transcription-polymerase chain reaction (qRT-PCR) that chidamide combined with venetoclax synergistically promoted apoptosis in AML cell lines and primary cells by inhibiting activation of the PI3K/AKT pathway and JAK2/STAT3 pathway. We present the following article in accordance with the ARRIVE reporting checklist (available at https://dx.doi.org/10.21037/atm-21-5066).

\section{Methods}

\section{Cell lines, primary tumor cells, and reagents}

The AML cell lines OCI-AML3, THP-1, MV4;11, and MOLM13 were purchased from the American Type Culture Collection (Manassas, VA, USA), and cultured in RPMI-1640 with $10 \%$ fetal bovine serum and penicillin (100 units/mL)/streptomycin $(100 \mu \mathrm{g} / \mathrm{mL})$. All cell lines were cultured in an incubator with $95 \%$ humidity at $37.5{ }^{\circ} \mathrm{C}$ with an atmosphere of $5 \% \mathrm{CO}_{2}$. Primary AML cells, collected before chemotherapy, were isolated from peripheral blood containing $>50 \%$ blasts. Venetoclax powder was purchased from the Selleck Company (USA) and dissolved in DMSO at a concentration of $10 \mathrm{mM}$, while chidamide was received as a gift from Shenzhen Microchip Biotechnology Co., Ltd. and dissolved in dimethyl sulfoxide 
(DMSO) at a concentration of $50 \mathrm{mM}$.

\section{Cell viability assay}

Cells were plated in 96-well plates (5,000 cells/well) and treated with different doses of venetoclax and chidamide for 24, 48, and $72 \mathrm{~h}$. At different time points, the cell number was measured using a Cell Counting Kit-8 (CCK8) proliferation assay kit (Tongren Institute of Chemistry, Japan). Ten microliters of CCK-8 solution were added to each well of the plate, and after incubation for $2 \mathrm{~h}$ at $37^{\circ} \mathrm{C}$, the plates were measured at $450 \mathrm{~nm}$ using a microplate reader (Biotech, NY, USA). The concentration of chidamide is $0.1-5 \mu \mathrm{M}$, and the concentration of venetoclax is $1 \mathrm{nM}-10 \mu \mathrm{M}$.

\section{Flow cytometry analysis}

\section{Apoptosis}

The AML cell lines were incubated with venetoclax and chidamide alone or in combination for 8 and $24 \mathrm{~h}$, and apoptosis was measured using Alexa Fluor 647-conjugated annexin $\mathrm{V}$ and propidium iodide (PI) (China Nanjing Kaiji Company) according to the manufacturer's instructions. Briefly, cells were harvested, washed twice with phosphatebuffered saline, and resuspended in $200 \mu \mathrm{L}$ of binding buffer before being incubated with $2 \mu \mathrm{L}$ Alexa Fluor 647-conjugated annexin $\mathrm{V}$ and $1 \mu \mathrm{L}$ PI for $10 \mathrm{~min}$. Apoptosis was analyzed using a Navios flow cytometer (Beckman Coulter, Brea CA, USA), and the results are expressed as the percentage of annexin $\mathrm{V}^{+}$cells. AML cell line experiments were performed three independent times in triplicate, and the data presented are from one representative experiment, while the patient sample experiments were performed once in triplicate due to the limited sample availability. The combination index (CI) values were determined using CompuSyn software where $\mathrm{CI}<1, \mathrm{CI}=1$, and $\mathrm{CI}>1$ indicated synergistic, additive, and antagonistic effects, respectively. The concentration of chidamide is $1-50 \mu \mathrm{M}$, and the concentration of venetoclax is $0.5 \mathrm{nM}-2 \mu \mathrm{M}$. All procedures performed in this study involving human participants were in accordance with the Declaration of Helsinki (as revised in 2013). The study was approved by the Medical Ethics Committee of The Affiliated Cancer Hospital of Zhengzhou University (approval No. 2021-KY-0170-001) and informed consent was taken from all the patients.

\section{Cell cycle analysis}

The cells were treated with venetoclax alone or combined with chidamide for $72 \mathrm{~h}$, then collected and washed with PBS and fixed overnight in $75 \%$ ice-cold ethanol at $4{ }^{\circ} \mathrm{C}$. The fixed cells were harvested, stained with PI/RNase (BD Pharmingen, San Diego, CA, USA), and incubated in the dark at room temperature for 15 min after being washed with PBS. The DNA content was analyzed by flow cytometry, and ModFit software (Verity Software House, Inc., Topsham, ME, USA) was used for data analysis. The concentration of chidamide is $1-2 \mu \mathrm{M}$, and the concentration of venetoclax is $0.5 \mathrm{nM}-0.5 \mu \mathrm{M}$.

\section{Analysis of the mitochondrial membrane potential}

Optimal concentrations of venetoclax and chidamide in the OCI-AML3, THP-1, MV4;11, and MOLM13 cell lines were determined according to the results of the above apoptosis assays. AML cells were then treated with venetoclax and chidamide alone or in combination for $24 \mathrm{~h}$, and JC-1, a cationic lipid fluorescent dye, was then used to stain the cells. During the process of apoptosis, the mitochondrial transmembrane potential decreases, JC-1 exists in the cytoplasm in the form of monomers, and the number of polymers decreases. Flow cytometry was used to determine whether JC-1 existed in the form of monomers or polymers to detect changes in the mitochondrial transmembrane potential. The concentration of chidamide is $5 \mu \mathrm{M}$, and the concentration of venetoclax is $1 \mathrm{nM}-0.5 \mu \mathrm{M}$.

\section{qRT-PCR assays}

Total cellular RNA was extracted from cells using TRIzol reagent (Invitrogen Life Technologies, Carlsbad, CA, USA) according to the manufacturer's protocol. RNA was eluted with RNase-free water, quantified at an absorbance of $260 / 280 \mathrm{~nm}$, and used for reverse transcription reactions, and total mRNA was reverse transcribed into cDNA using an RT reagent kit (TaKaRa, Dalian, China). Primer sequences of qRT-PCR are shown in Table 1 , and $\beta$-actin was used as an internal standard. qRT-PCR was performed with Fast Start Universal SYBR Green Master Mix (ROX) (Roche, Germany) following the instructions of the supplier and under the following conditions: one cycle at $94^{\circ} \mathrm{C}$ for $10 \mathrm{~min}, 40$ cycles at $94^{\circ} \mathrm{C}$ for $10 \mathrm{~s}, 60^{\circ} \mathrm{C}$ for $30 \mathrm{~s}$, and one cycle at $72{ }^{\circ} \mathrm{C}$ for $3 \mathrm{~min}$. The results were analyzed using the $2^{-\Delta \Delta C t}$ method, in which $\Delta \mathrm{Ct}=\mathrm{Ct}$ (target gene) $-\mathrm{Ct}$ (internal reference), and $\Delta \Delta \mathrm{Ct}=\Delta \mathrm{Ct}$ (sample) $-\Delta \mathrm{Ct}$ (control), and 
Table 1 Primer sequences of qRT-PCR

\begin{tabular}{|c|c|}
\hline Gene & Primer \\
\hline $\mathrm{Bcl}-2$ forward & 5'-CGACTTCGCCGAGATGTCCAG-3' \\
\hline $\mathrm{Bcl}-2$ reverse & 5'-CGGTTCAGGTACTCAGTCATCCAC-3' \\
\hline $\mathrm{Mcl}-1$ forward & 5'-GCCGCTGACGCCATCATGTC-3' \\
\hline $\mathrm{Mcl}-1$ reverse & 5'-СААСTCGTCСТССТССТССTVTG-3' \\
\hline$B c /-x /$ forward & 5'-GTGCGTGGAGAGCGTAGACAAG-3' \\
\hline$B c l-x /$ reverse & 5'-AGAGCGAGCCCAGCAGAACC-3' \\
\hline Jak2 forward & 5'-CGGTGGAATTCAGTGGTCAAGAGG-3' \\
\hline Jak2 reverse & 5'-CGGCTGGAGGTGCTACTTCTTTAC-3' \\
\hline STAT3 forward & 5'-AGGGAGCAGAGATGTGGGAATGG-3' \\
\hline STAT3 reverse & 5'-TCGCTTGGTGGTGGAGGAGAAC-3' \\
\hline sOCS3 forward & 5'-GGTCACCCACAGCAAGTTTCCC-3' \\
\hline SOCS3 reverse & 5'-CACCAGCTTGAGCACGCAGTC-3' \\
\hline$A K T$ forward & 5'-GTGGCTATTGTGAAGGAGGGTTGG-3' \\
\hline$A K T$ reverse & 5'-GCAGGCAGCGGATGATGAAGG-3' \\
\hline$H D A C 1$ forward & 5'-TATCGCCCTCACAAAGCCAATGC-3' \\
\hline$H D A C 1$ reverse & 5'-CTTGCCACAGAACCACCAGTAGAC-3' \\
\hline P21 forward & 5'-CCTGTCACTGTCTTGTACСCT-3' \\
\hline$P 21$ reverse & 5'-GCGTTTGGAGTGGTAGAAATCT-3' \\
\hline CDK2 forward & 5'-GGCCATCAAGCTAGCAGACT-3' \\
\hline CDK2 reverse & 5'-GAATCTCCAGGGAATAGGGC-3' \\
\hline C-myc forward & 5'-CCACAGCAAACCTCCTCACAG-3' \\
\hline C-myc reverse & 5'-GCAGGATAGTCCTTCCGAGTG-3' \\
\hline$\beta$-actin forward & 5'-CGCTGCGCTGGTCGTCGACA-3' \\
\hline$\beta$-actin reverse & 5'-GTCACGCACGATTTCCCGCT-3' \\
\hline
\end{tabular}

each sample was measured in triplicate. The concentration of chidamide is $1-50 \mu \mathrm{M}$, and the concentration of venetoclax is $0.5 \mathrm{nM}-0.5 \mu \mathrm{M}$.

\section{Western blot analysis}

After treating cells with venetoclax and chidamide for $24 \mathrm{~h}$, cultured cells were harvested, washed with PBS and lysed with ice-cold lysis buffer. The protein lysates were clarified by centrifugation at $14,000 \mathrm{~g}$ for $15 \mathrm{~min}$ at $4^{\circ} \mathrm{C}$, and the supernatant was collected. The protein level in each sample was quantified by a bicinchoninic acid (BCA) assay (Pierce). Equal amounts of proteins were separated by SDS-PAGE and then electrotransferred onto PVDF membranes (Millipore), which were then blocked with 5\% skim milk and incubated with primary antibodies at $4{ }^{\circ} \mathrm{C}$ overnight in TBS-T (10 mM Tris-HCl, $\mathrm{pH} 8,150 \mathrm{mM} \mathrm{NaCl}$, and $0.1 \%$ Tween 20). Primary antibodies against the following proteins were used: $\gamma-\mathrm{H} 2 \mathrm{AX}$, PARP, caspase 3, Bcl-2, Mcl1, Bim, Bax, Bak, AKT, P-AKT, SOCS3, P-JAK2, and P-STAT3, and $\beta$-actin (Cell Signaling, Herts, UK) was used as a loading control. The blots were washed, exposed for $1 \mathrm{~h}$ to the corresponding horseradish peroxidase (HRP)conjugated secondary antibodies, and finally detected by chemiluminescence reagents (Millipore, Billerica, MA, USA). The target protein bands were then visualized using enhanced chemiluminescence (ECL) and exposed to X-ray film. Immunoreactive proteins were visualized using an Odyssey Infrared Imaging System (Li-Cor, Lincoln, NE, USA), as described by the manufacturer, and densitometry measurements were made using an Odyssey V3.0 (Li-Cor) and normalized to $\beta$-actin. The concentration of chidamide is $1 \mu \mathrm{M}-50 \mu \mathrm{M}$, and the concentration of venetoclax is $0.5 \mathrm{nM}-1 \mu \mathrm{M}$.

\section{HDAC inbibitory activity of chidamide}

The HDAC1 gene level was detected by qRT-PCR, and $\mathrm{HDAC} 1$ protein, acetylated histone $\mathrm{H} 3$, and histone $\mathrm{H} 4$ levels were detected by Western blot to determine the ability of chidamide to inhibit histone deacetylation.

\section{In vivo experiment}

To reflect the tumor load by detecting the fluorescence intensity, MOLM13 cells expressing luciferase need to be constructed first. Construction of firefly luciferaseexpressing MOLM13 cells-vector expressing luciferase prenti-CMV-Puro-Luc (w168-1) was a gift from Eric campeau \& Paul Kaufman (addgene plasma \# 17477; http://n2t.net/addgene: 17477; RRID: Addgene_17477). Lentivirus packaging plasmid was transfected into $293 \mathrm{~T}$ cells. The supernatant was collected and concentrated 48 and $72 \mathrm{~h}$ after transfection to obtain lentivirus. Lentivirus transduced MOLM13 cells in logarithmic growth stage. MOLM13 cells transfected with luciferase were injected $\left(5 \times 10^{6}\right.$ cells $)$ via the tail vein in mice which were 20 gram, 5-6 weeks and female with severe immunodeficiency (NOD/SCID). Through literature review $(30,31)$, we determined the experimental concentrations of venetoclax and chidamide. In vivo imaging was performed 4 days 
after the injection to confirm the successful establishment of an AML xenotransplantation mouse model. The mice were randomly divided into groups with nine mice in each group, and treated with venetoclax $(100 \mathrm{mg} / \mathrm{kg}$, intragastric administration, QD) and chidamide $(25 \mathrm{mg} / \mathrm{kg}$, intragastric administration, QD) alone or in combination. The untreated group was considered the control, and the tumor growth was observed twice per week. The mice were anesthetized with isoflurane gas, and the substrate fluorescein sodium salt (concentration: $15 \mathrm{mg} / \mathrm{mL}$ ) was injected intraperitoneally. The mice were imaged in vivo by small animal viviscreator. The average fluorescence intensity of three parts of each mouse was taken as the final fluorescence intensity to indirectly reflect the tumor load of the mouse. Three mice in each group were sacrificed on the 10th day of treatment, the liver and spleen were collected for immunohistochemical staining with a human CD45 antibody (hCD45), and the expression of $\mathrm{CD} 45^{+} \mathrm{CD} 33^{+}$cells in the bone marrow was analyzed by flow cytometry. The survival time of the remaining six mice in each group was observed, and the survival curve of tumor-bearing mice was generated. The weight of the mice in the four groups was measured every other day, and a weight curve was generated after 14 days of continuous monitoring. Experiments were performed under a project license (approval No. 20200320) granted by Ethics Review Committee of life sciences, Zhengzhou University, in compliance with Zhengzhou University national or institutional guidelines for the care and use of animals. A protocol was prepared before the study without registration.

\section{Statistical analysis}

Each experiment was performed at least three times, and the data are presented as the mean \pm standard deviation for the indicated number of separate experiments. A $t$-test was used to compare the mean of each group with that of the control group in experiments. All analysis was performed with SPSS 22.0 System, and the results were considered significant if the $\mathrm{P}$ value was less than 0.05 .

\section{Results}

\section{Venetoclax and chidamide synergistically inbibit cell proliferation}

To determine the effect of venetoclax and chidamide on proliferation, we first examined the proliferation of OCI-
AML3, THP-1, MV4;11, and MOLM13 cells in response to venetoclax and chidamide at various concentrations using a CCK-8 assay. The half-maximal inhibitory concentration (IC50) of venetoclax and chidamide for the cell lines are depicted in Figure 1A-1C, which shows measurements were performed in three independent experiments after 24, 48, and $72 \mathrm{~h}$ of exposure. The results show venetoclax and chidamide caused growth arrest in the OCI-AML3, THP1, MV4;11, and MOLM13 cell lines in a concentration- and time-dependent manner (Figure 1D,1E). We believe that an IC50 $>1 \mu \mathrm{M}$ is indicative of a relatively resistant cell line, which suggests OCI-AML 3 and THP-1 cells are relatively resistant to venetoclax and chidamide, and MV4;11 and MOLM13 cells are relatively sensitive to venetoclax and chidamide.

\section{Venetoclax and chidamide synergistically promote apoptosis in AML cells}

According to previous reports, both venetoclax and chidamide can induce apoptosis. Therefore, we measured the apoptosis rate in response to monotherapy and combination treatment with flow cytometry. Figure $2 A-2 C$ shows that venetoclax and chidamide induced apoptosis in four AML cell lines and primary AML cells in a concentration- and time-dependent manner, and their combination had a synergistic effect on apoptosis (CI $<1$ ). With patient consent, the bone marrow (primitive or immature cells $>50 \%$ ) of five newly diagnosed AML patients (AML\#01-AML\#05) was extracted to isolate mononuclear cells, and their clinical characteristics are described in Table 2. Additionally, we detected changes in apoptosisrelated genes at the mRNA level with qRT-PCR, and as shown in Figure 2D, the expression of the antiapoptotic gene Bcl-2 was decreased in the venetoclax and chidamide monotherapy groups, and further decreased in the combination therapy group, with the difference being statistically significant $(\mathrm{P}<0.05)$. The expression of the antiapoptotic gene Mcl-1 was increased in the venetoclax monotherapy group, decreased in the chidamide monotherapy group, and further decreased in the combination therapy group, and the difference was statistically significant $(\mathrm{P}<0.05)$. The expression of the antiapoptotic gene Bcl-x1 was significantly decreased in the monotherapy groups and the combination therapy group $(\mathrm{P}<0.05)$.

In addition, we also examined apoptosis-related proteins in the mitochondrial pathway and Bcl-2 family apoptosis 
A

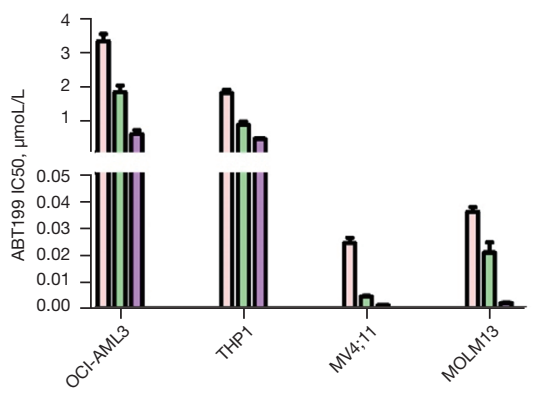

B

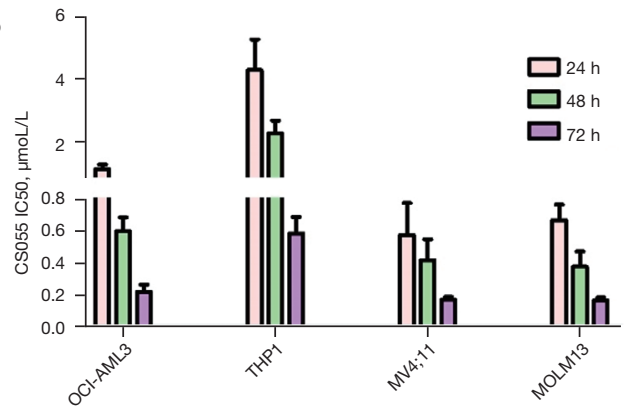

C

\begin{tabular}{|c|c|c|c|c|c|c|c|}
\hline \multirow{2}{*}{ Cell lines } & \multicolumn{3}{|c|}{ ABT199 IC50, $\mu \mathrm{M}$} & \multicolumn{3}{|c|}{ CS055 IC50, $\mu \mathrm{M}$} & IC50, $\mu \mathrm{I}$ \\
\hline & $24 \mathrm{~h}$ & $48 \mathrm{~h}$ & $72 \mathrm{~h}$ & $24 \mathrm{~h}$ & $48 \mathrm{~h}$ & $72 \mathrm{~h}$ & $0-0.01$ \\
\hline \multirow{2}{*}{ OCI-AML3 } & \multirow{2}{*}{3.291} & \multirow{2}{*}{1.802} & \multirow{2}{*}{0.493} & \multirow{2}{*}{1.043} & \multirow{2}{*}{0.591} & \multirow{2}{*}{0.213} & $0.01-0.1$ \\
\hline & & & & & & & $0.1-1$ \\
\hline THP1 & 1.771 & 0.889 & 0.401 & 4.243 & 2.203 & 0.577 & $1-5$ \\
\hline MV4;11 & 0.023 & 0.004 & 0.0009 & 0.567 & 0.411 & 0.163 & \\
\hline MOLM13 & 0.036 & 0.026 & 0.0005 & 0.661 & 0.370 & 0.162 & \\
\hline
\end{tabular}

D
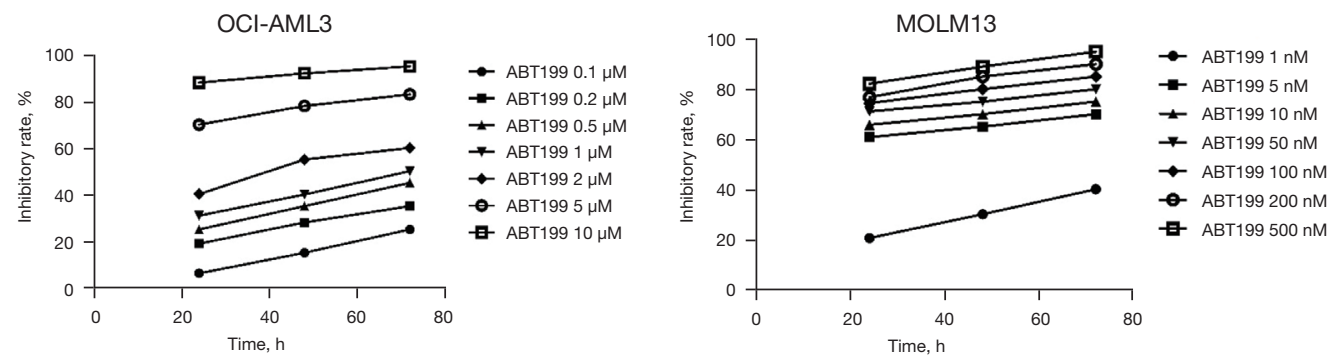

$\mathrm{E}$
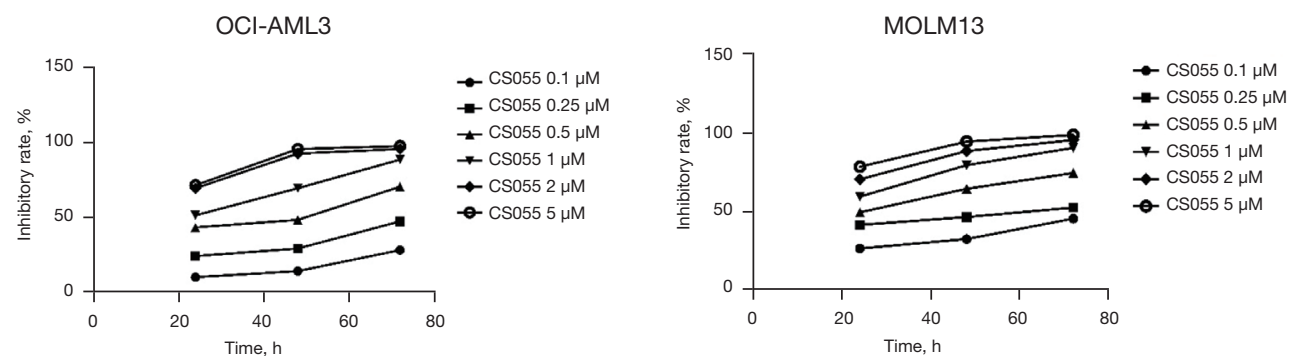

Figure 1 The IC50 values of ABT199 (venetoclax) and CS055 (chidamide) on AML cell lines (OCI-AML3, THP-1, MV4;11, and MOLM13) at 24, 48 and $72 \mathrm{~h}$, and the proliferation inhibition curves of different concentrations of ABT199 and CS055 on OCI-AML3 and MOLM13 cell lines at 24, 48 and $72 \mathrm{~h}$. (A) IC50 values of four AML cell lines treated with ABT199 for 24, 48, and $72 \mathrm{~h}$. (B) IC50 values of four AML cell lines treated with CS055 for 24, 48, and $72 \mathrm{~h}$. (C) IC50 values of the two drugs in four AML cell lines at 24, 48, and $72 \mathrm{~h}$. (D) The proliferation inhibition curve of ABT199 for OCI-AML3 and MOLM13 at 24, 48, and $72 \mathrm{~h}$. (E) The proliferation inhibition curve of CS055 for OCI-AML3 and MOLM13 at 24, 48, and 72 h. AML, acute myeloid leukemia. 

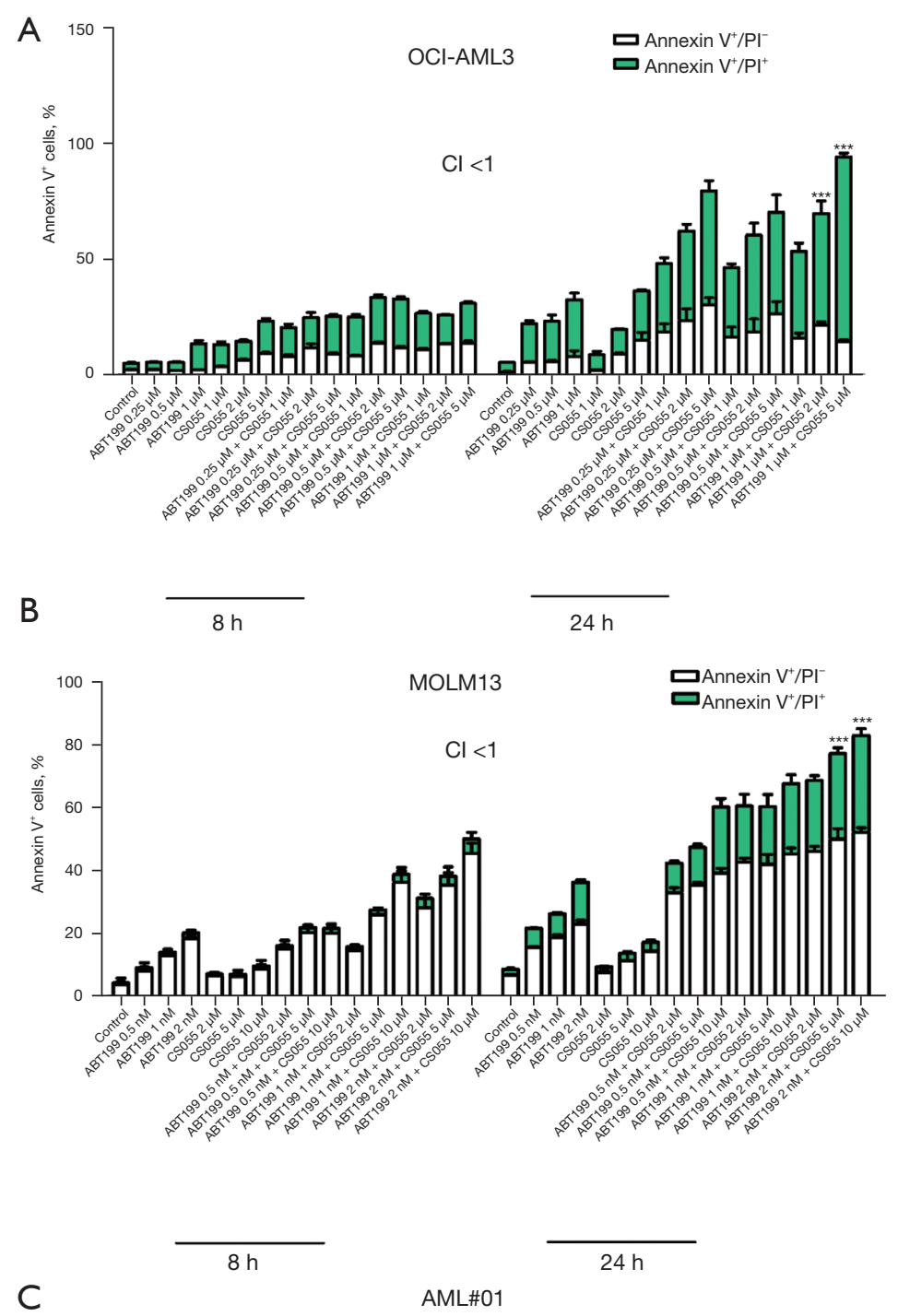

C

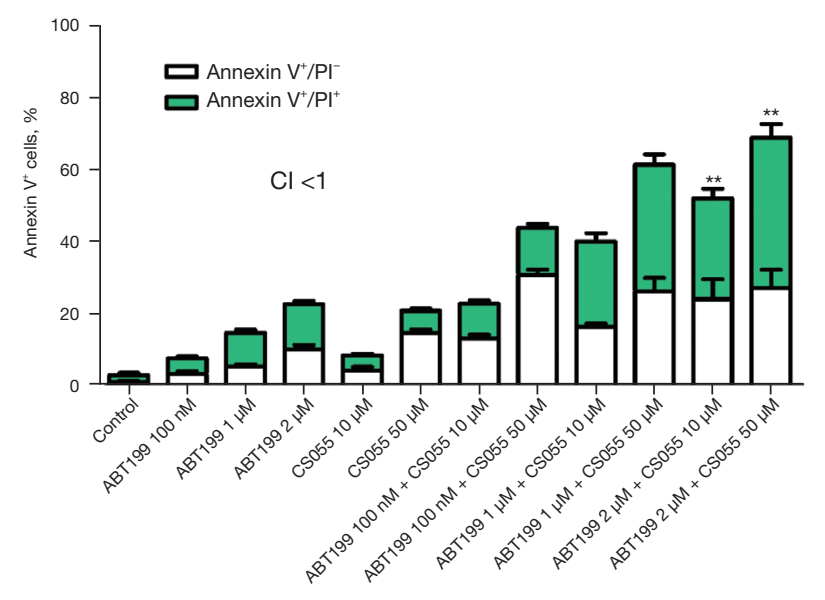



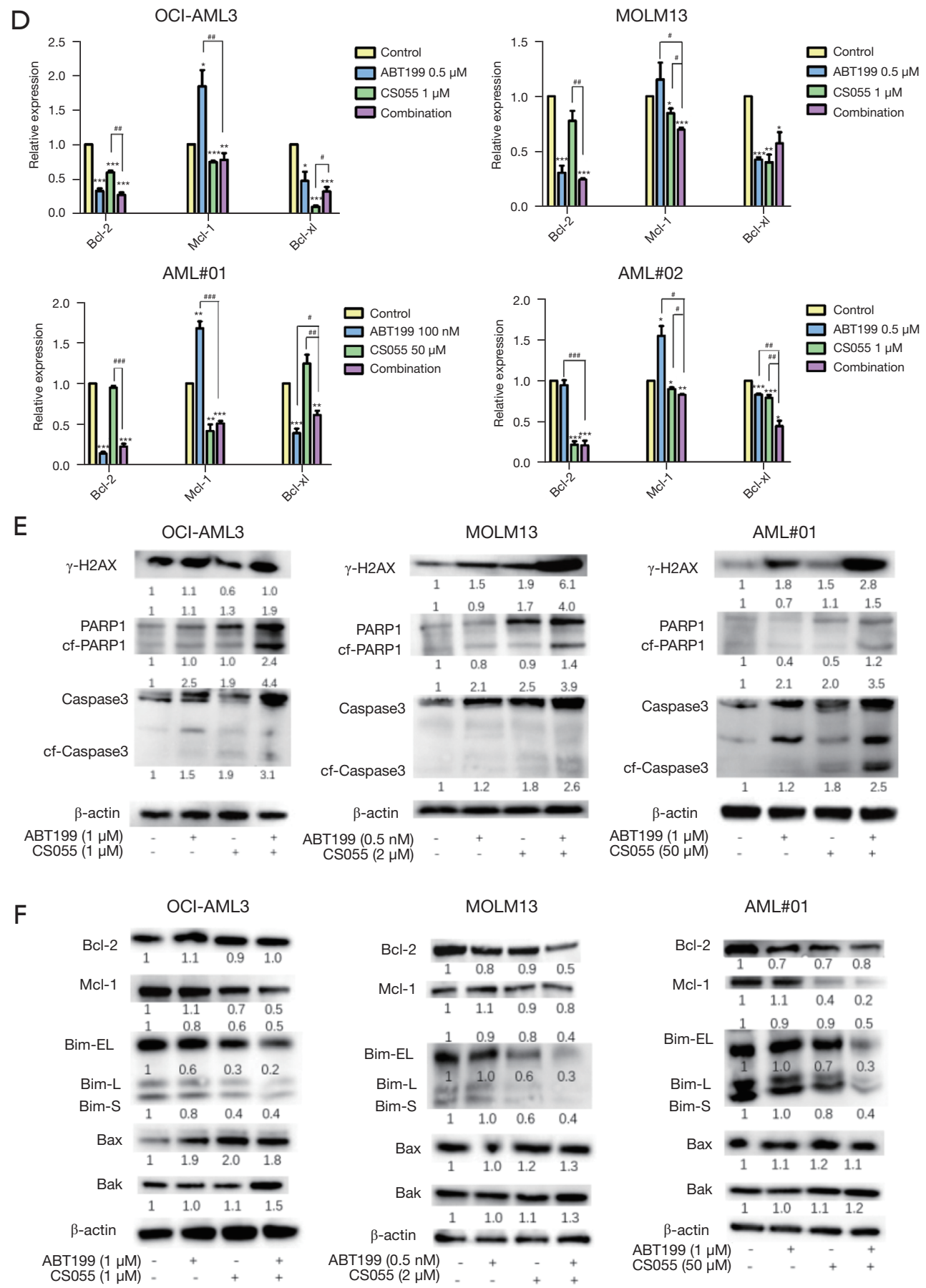

Figure 2 ABT199 (Venetoclax) and CS055(chidamide) single and combined effects on the apoptosis rate of AML cell line OCI-AML3 and MOLM13 at 8 and 24 h, and the changes of apoptosis related genes and proteins at 24 h. (A) Apoptosis histograms of the OCI-AML3 treated with ABT199 or CS055 alone or in combination for 8 and 24 h. (B) Apoptosis histograms of the OCI-AML3 treated with ABT199 or CS055 alone or in combination for 8 and 24 h. (C) ABT199 and CS055 monotherapy and combination treatment of primary AML cells. (D) Changes in apoptosis-related proteins detected by qRT-PCR. (E) Changes in apoptosis-related proteins detected by Western blot. (F) Changes in Bcl-2 family apoptotic regulatory proteins detected by Western blot. * $\mathrm{P}<0.05 ;{ }^{* *}, \mathrm{P}<0.01 ;{ }^{* * *}, \mathrm{P}<0.001$, compared with the control group. ", $\mathrm{P}<0.05 ;{ }^{\#}, \mathrm{P}<0.01 ;{ }^{\# \#}, \mathrm{P}<0.001$. AML, acute myeloid leukemia. 
Table 2 Clinical characteristics of AML patients

\begin{tabular}{|c|c|c|c|c|c|c|c|c|}
\hline $\begin{array}{l}\text { Case } \\
\text { number }\end{array}$ & Gender & $\begin{array}{c}\text { Age } \\
\text { (years) }\end{array}$ & Disease status & $\begin{array}{l}\text { FAB } \\
\text { type }\end{array}$ & $\begin{array}{c}\text { Prime/immature } \\
\text { cells }\end{array}$ & Molecular mutation & Fusion gene & Chromosome \\
\hline \#01 & Male & 22 & Newly diagnosed & M2b & $86.8 \%$ & c-kit/ABCB1 & AML1-ETO & $45, X Y, t(8 ; 21)(q 22 ; q 22)[20]$ \\
\hline$\# 03$ & Male & 61 & Newly diagnosed & M2b & $65 \%$ & C-kit/ABCB1 & AML1-ETO & $45, X Y, t(8 ; 21)(q 22 ; q 22)[20]$ \\
\hline \#05 & Female & 45 & Newly diagnosed & M1 & $96 \%$ & $\begin{array}{l}\text { FLT3-ITD, NPM1, IDH2, } \\
\text { TET2, ABCB1 }\end{array}$ & Negative & $46, X X[16]$ \\
\hline
\end{tabular}

AML, acute myeloid leukemia.

regulatory proteins by Western blot. Figure $2 E$ shows that the expression of the apoptosis-related protein cfCaspase- 3 in the monotherapy groups was higher than that in the control group, and this effect was further enhanced in the combination therapy group. The protein expression of cf-PARP1, whose cleavage is considered an important indicator of apoptosis and caspase 3 activation, was significantly higher in the combination group than in the control group. The expression levels of $\gamma$-H2AX protein in the two monotherapy groups were higher than those in the control group, and the effect was further enhanced in the combination therapy group. Moreover, Figure $2 F$ shows that the antiapoptotic protein Bcl-2 was decreased in the chidamide monotherapy group and the combination therapy group. The antiapoptotic protein Mcl-1 was increased by venetoclax monotherapy, decreased by chidamide monotherapy, and further decreased by combination therapy, while the expression of the "BH3only" proapoptotic protein Bim in the venetoclax and chidamide monotherapy groups was decreased, and this effect was further enhanced in the combination therapy group. The expression of the proapoptotic protein Bax/Bak in the monotherapy groups and the combination therapy group was higher than that in the control group.

\section{Venetoclax and chidamide synergistically decrease the mitochondrial membrane potential}

As shown in Figure 3, venetoclax and chidamide monotherapy reduced the mitochondrial membrane potential of AML cells in the four AML cell lines. In the combination therapy group, the effect was significantly enhanced, and the mitochondrial membrane potential was significantly different from that in the monotherapy group and control group.

\section{Venetoclax and chidamide synergistically induce cell cycle arrest}

As shown in Figure 4A, venetoclax and chidamide blocked the cell cycle of the AML cell lines in the G1 phase and of primary cells in the $S$ phase, which inhibited proliferation, and the blocking effect was further enhanced by combination of the two drugs. Figure $4 B$ shows that the apoptosis inhibited gene p21 was upregulated and that the downstream factors CDK2 and myc were downregulated, as detected by qRT-PCR, resulting in cell cycle arrest. Figure $4 C$ shows that apoptosis inhibitory protein $\mathrm{p} 21$ was upregulated, and the downstream factors CDK2 and c-myc were downregulated, as detected by Western blot, resulting in cell cycle arrest.

\section{Transcriptome sequencing and cluster analysis showed that the differentially expressed genes were enriched in the PI3K-AKT and $7 A K 2 / S T A T 3$ pathways in the venetoclax monotherapy group vs the combination therapy group}

As shown in Figure $5 A-5 C$, the number of differentially expressed genes in the venetoclax monotherapy and combination groups was examined after $24 \mathrm{~h}$ of treatment, where $\mathrm{B}$ shows the venetoclax monotherapy group, and $\mathrm{E}$ shows the venetoclax + chidamide group. After $24 \mathrm{~h}$ of drug treatment, the differentially expressed genes in each group were clustered. By counting the number of differentially expressed genes in different layers and the levels of KEGG pathways, we determined which metabolic pathways and signaling pathways they were mainly involved in. Cluster analysis of transcriptome sequencing showed that after 


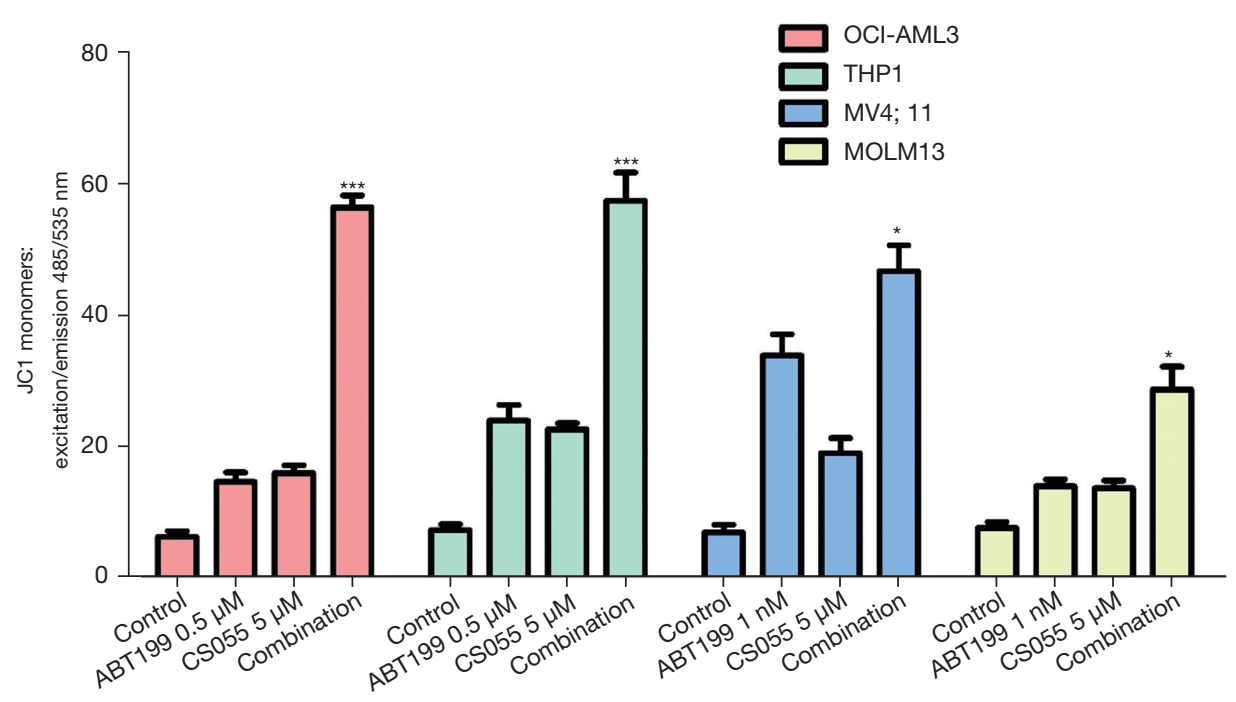

Figure 3 Histogram of changes in the mitochondrial membrane potential in the OCI-AML3, THP1, MV4;11, and MOLM13 cell lines treated with ABT199 (venetoclax) and CS055 (chidamide). * $\mathrm{P}<0.05 ;{ }^{* * *}, \mathrm{P}<0.001$, compared with the control group.

venetoclax and chidamide were added to OCI-AML3 cells, the gene clustering of the venetoclax monotherapy group and negative control group was basically the same, and the expression levels of most genes changed after chidamide was added to the cells. We also found that in OCI-AML3 cells, genes related to the PI3K-AKT pathway and JAK2/STAT3 were differentially expressed in the venetoclax monotherapy group and venetoclax + chidamide group.

\section{qRT-PCR confirmed the PI3K-AKT and JAK2/STAT3 pathways were inbibited and the expression of HDAC1 increased}

We used qRT-PCR to analyze the mRNA expression levels of the above two pathways and HDAC1, and the results are shown in Figure 5D. (I) In the PI3K-AKT pathway, the expression of AKT was downregulated, upregulating the expression of the downstream factor $\mathrm{p} 21$ and downregulating the expression of CDK2 and c-myc $(\mathrm{P}<0.05)$. (II) For the JAK2/STAT3 pathway, the expression of SOCS3 was upregulated, downregulating the expression of the downstream factors JAK2 and STAT3, and the difference was statistically significant $(\mathrm{P}<0.05)$. (III) For HDAC1, the expression of HDAC1 was upregulated in the chidamide group and the combination group, and the difference was statistically significant $(\mathrm{P}<0.05)$.

\section{Western blot showed that the PI3K-AKT and $\mathcal{A} A K 2 /$ STAT3 pathways were inbibited and the expression of HDAC1 increased}

Western blot analysis results showed the PI3K-AKT and JAK2/STAT3 pathways were both inhibited, and the expression of HDAC1 was upregulated, with the results shown in Figure $5 E$ (1). Chidamide inhibited the activation of the PI3K-AKT pathway by downregulating the expression of $\mathrm{P}-\mathrm{AKT}$ protein, upregulating the expression of the downstream factor apoptosis-inhibited protein p21, downregulating the expression of CDK2 and c-myc, and resulting in cell cycle arrest (2). Further, chidamide inhibited the activation of the JAK2/STAT3 signaling pathway by upregulating SOCS3 protein expression and downregulating P-JAK2 protein expression. Thereby, it upregulated downstream p21 protein expression, inhibited downstream CDK2 and c-myc protein expression of STAT3, caused cell cycle arrest, and inhibited proliferation. At the same time, it inhibited the expression of Bcl-2, Bcl$\mathrm{xl}$, and Mcl-1, and promoted apoptosis (3). In regard to the HDAC1 protein level, the expression of HDAC1, acetyl-H3, and $\mathrm{H} 4$ was upregulated, resulting in the prevention of histone deacetylation and an increased degree of histone acetylation, which caused cell proliferation to be inhibited by upregulating p21 protein levels. 

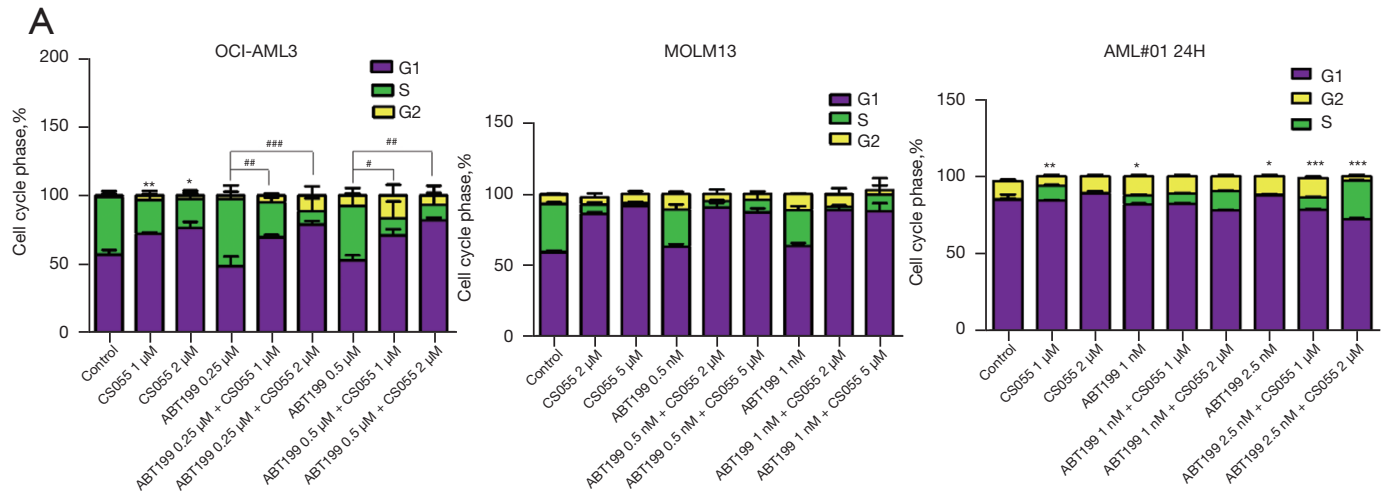

B
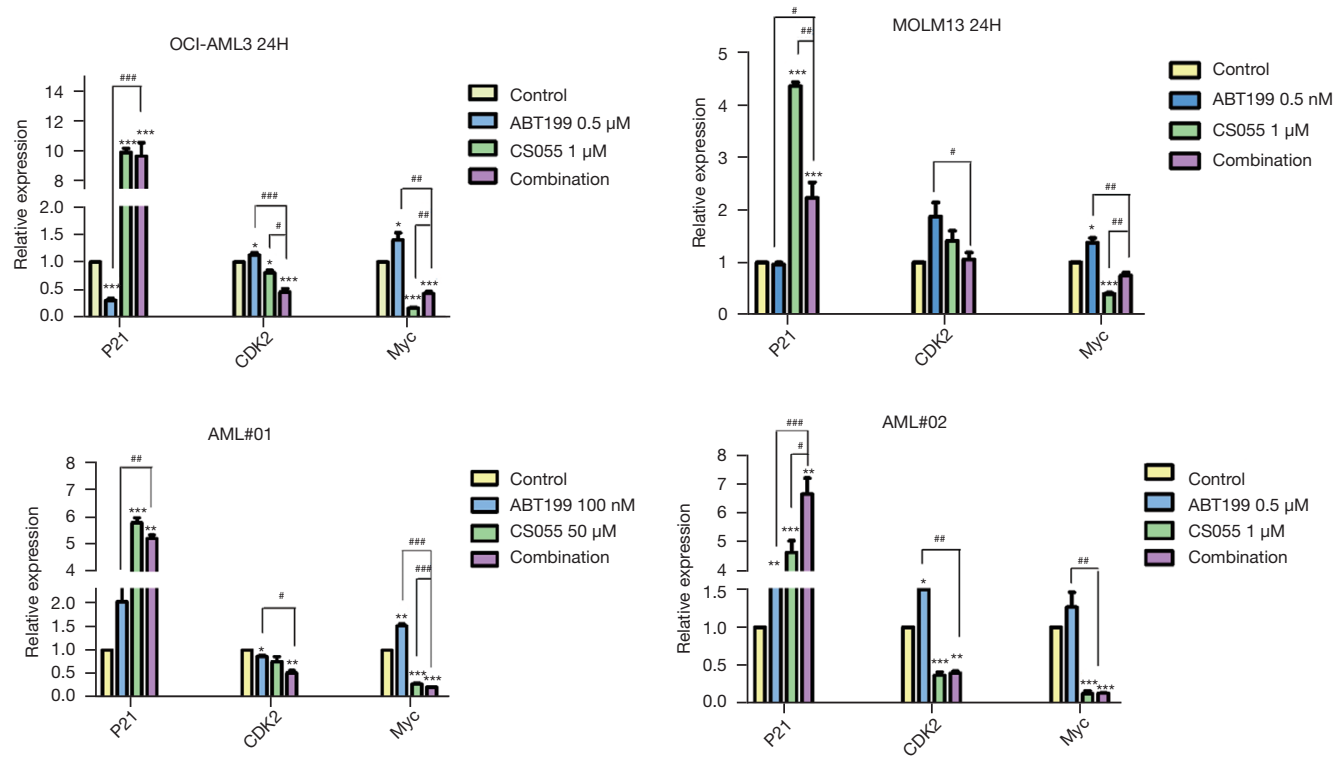

C
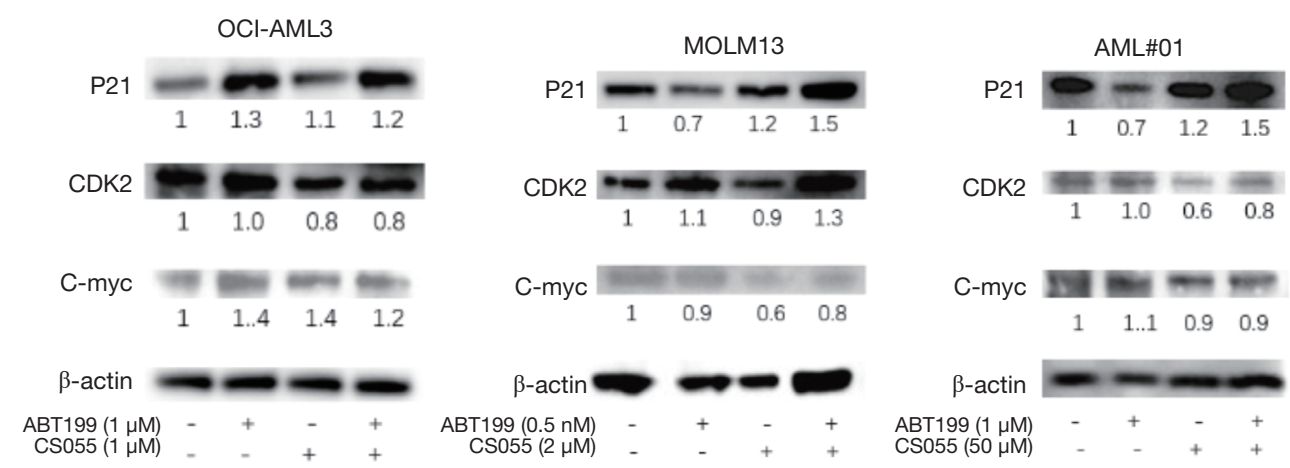

CDK2

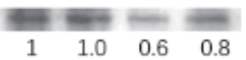

C-myc

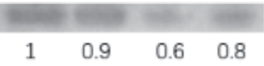

C-myc
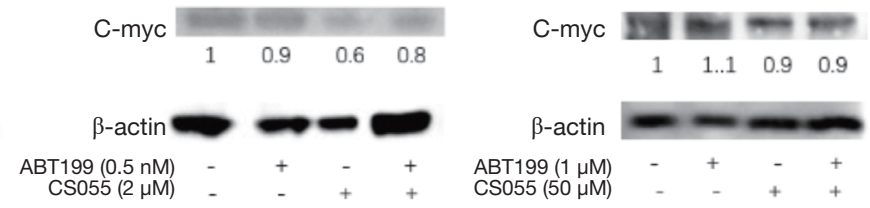

Figure 4 Cell cycle histogram, cycle related genes and protein changes of AML cell lines (OCI-AML3, MOLM13) and primary cells treated with ABT199 (venetoclax) and CS055 (chidamide) for 24 h. (A) Cell cycle analysis after ABT199 and CS055 monotherapy and combination therapy in different AML cell lines and primary AML cells with flow cytometry. (B) Changes in cell cycle-related genes before and after treatment, as detected by qRT-PCR. The data were statistically analyzed with a $t$-test. (C) Changes in cell cycle-related proteins before and after treatment, as detected by Western blot. ${ }^{*}, \mathrm{P}<0.05$; ${ }^{* *}, \mathrm{P}<0.01$; ${ }^{* *}, \mathrm{P}<0.001$, compared with the control group. ${ }^{*}, \mathrm{P}<0.05$; ${ }^{\# \#,} \mathrm{P}<0.01$; ${ }^{\# \# \#}$, $\mathrm{P}<0.001$. AML, acute myeloid leukemia. 

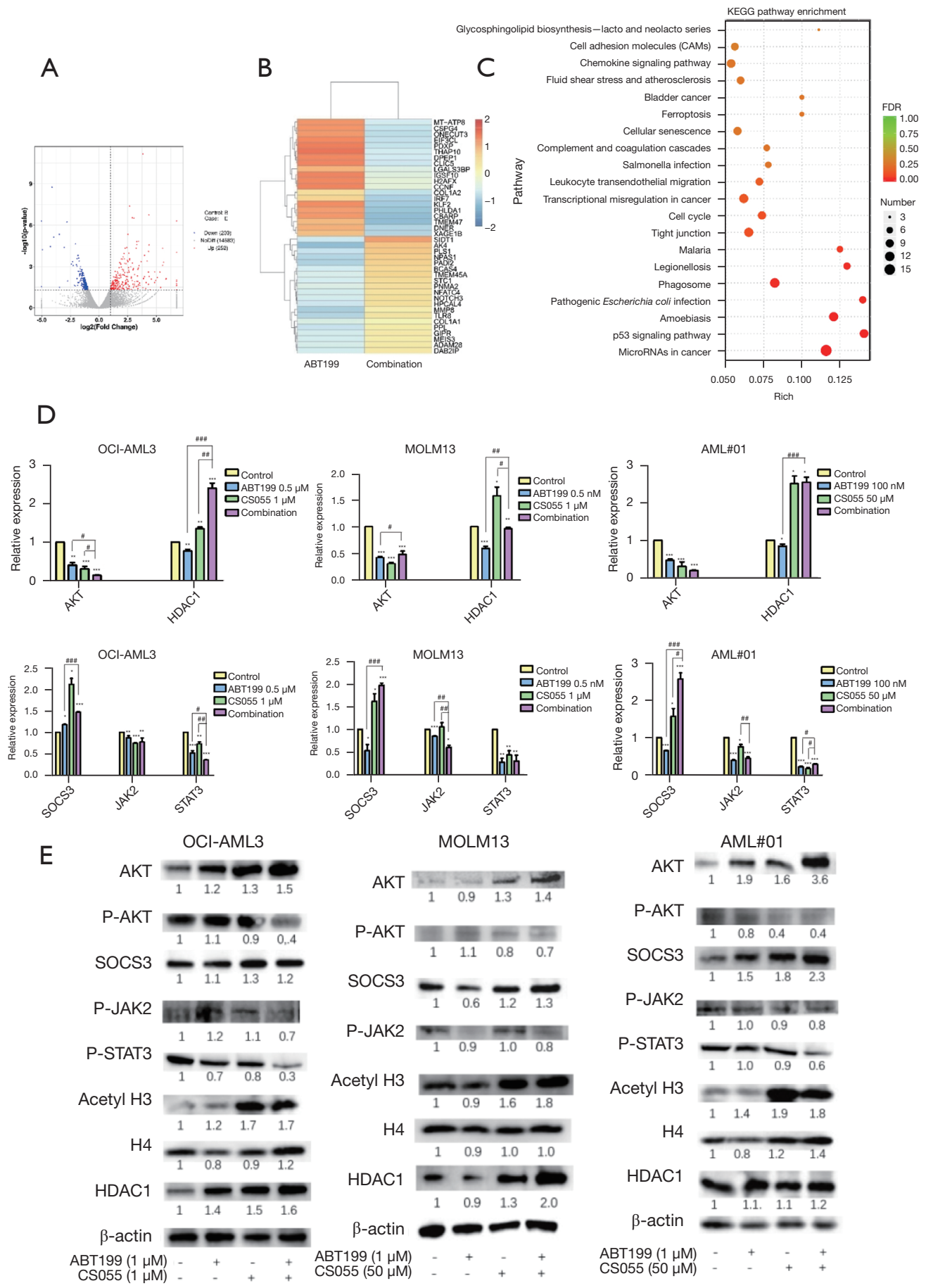

Figure 5 Differential expression gene analysis and KEGG pathway enrichment results of transcriptome sequencing samples, as well as relevant results of signal pathway verification by qRT-PCR and Western blot. (A) Volcano map of differentially expressed genes. (B) Genes with the most obvious differences in expression. (C) Enrichment analysis of KEGG pathways. (D) mRNA levels of AKT, HDAC1, SOCS3, JAK2, and STAT3 expressed in OCI-AML, MOLM13, and primary cells treated with ABT199 (venetoclax) and CS055 (chidamide) for 24 h, as analyzed by qRT-PCR. The data were statistically analyzed by a $t$-test. (E) PI3K-AKT and JAK2/STAT3 signaling pathway-related proteins and HDAC1 protein expression levels in the OCI-AML3 and MOLM13 cell lines and AML primary cells after 24 h of treatment with ABT199 and CS055. *, $\mathrm{P}<0.05 ;{ }^{* *}, \mathrm{P}<0.01{ }^{* * *}, \mathrm{P}<0.001$, compared with the control group. ${ }^{\#}, \mathrm{P}<0.05 ;{ }^{\# \#}, \mathrm{P}<0.01 ;{ }^{\# \# \#}, \mathrm{P}<0.001$. AML, acute myeloid leukemia. 


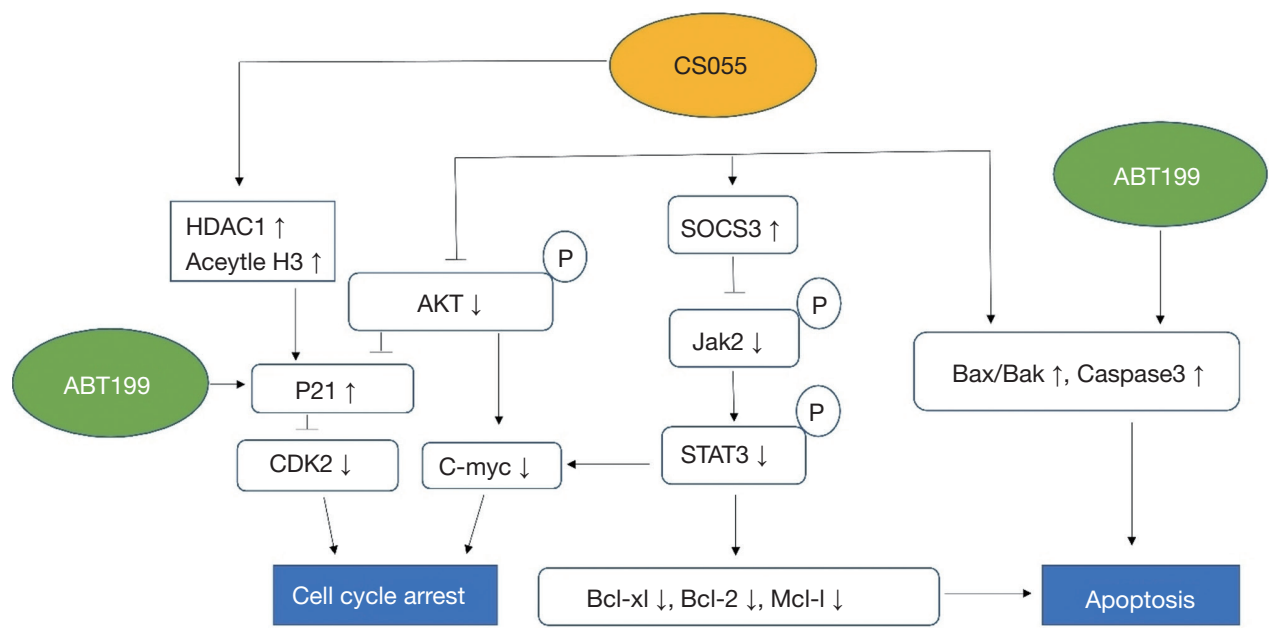

Figure 6 Signaling pathway of ABT199 (venetoclax) combined with CS055 (chidamide) in AML cells. AML, acute myeloid leukemia.

\section{Signaling pathways of venetoclax combined with chidamide in AML cells}

Finally, the signaling pathways of chidamide and venetoclax in AML cells are summarized in Figure 6.

\section{In vivo experiment}

Compared with the monotherapy groups and control group, the combination therapy group exhibited significantly inhibited disease progression and prolonged survival time in AML mice. The results show that: (I) venetoclax alone prolonged the survival time of mice $(\mathrm{P}<0.05)$, whereas chidamide had no significant effect. The survival time was further prolonged after venetoclax was combined with chidamide $(\mathrm{P}<0.01)$ (Figure $7 A)$. (II) Compared with the control group, the fluorescence intensity decreased in the chidamide group, but there was no significant difference. However, the fluorescence intensity decreased in the venetoclax group and further decreased in the combination group, and the difference was statistically significant $(\mathrm{P}<0.001)$ (Figure $7 B, 7 C)$, which showed venetoclax alone significantly inhibited the growth of tumors in AML xenograft mice, and this effect was further enhanced after combination with chidamide. (III) Flow cytometry showed that compared with the control group, the number of $\mathrm{CD} 45^{+} \mathrm{CD} 33^{+}$cells in the bone marrow of the monotherapy groups and the combination treatment group decreased, and the combination group had the lowest number, with the difference statistically significant $(\mathrm{P}<0.05)$ (Figure $7 D, 7 E)$. On the 14th day, the body weight of mice in the chidamide group and the control group was significantly lower than that in the venetoclax group and the combination group, and the difference was statistically significant $(\mathrm{P}<0.01)$ (Figure $7 F$ ) (4). On the 10th day of treatment, three mice in each group were sacrificed and dissected, and in vivo imaging showed that AML leukemia cells infiltrated the lungs, liver, spleen, kidneys, and femurs. The liver, spleen, and lung of mice in each group were then stained with $\mathrm{HE}$, immunohistochemical staining was performed with an hCD45 antibody, and the expression intensity of hCD45 was as follows: control group > chidamide group > venetoclax group > combination group (Figure $7 G, 7 H$ ).

\section{Discussion}

In this study, both venetoclax and chidamide inhibited the proliferation of AML cells in a time-dependent and concentration-dependent manner, which was consistent with previous reports (32). At the same time, we measured the apoptosis rate by flow cytometry and confirmed both drugs promoted the apoptosis of AML cell lines and primary cells in a time-dependent and concentration-dependent manner. The combination of the two further enhanced the effect of apoptosis promotion and had a synergistic effect (CI $>1)$. Venetoclax promotes apoptosis in AML cells mainly through the mitochondrial pathway. Kroemer et al. (33) reported that the mitochondrial transmembrane potential is necessary to maintain normal mitochondrial function. In the early stage of apoptosis, the mitochondrial permeability transition pore (PT pore) opens, which leads to a decrease 


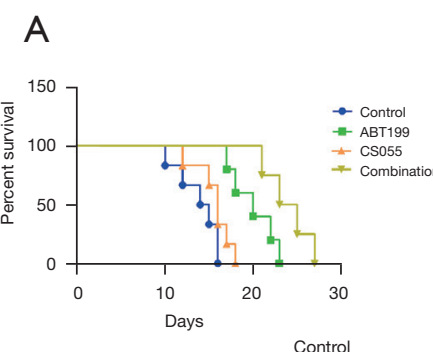

B

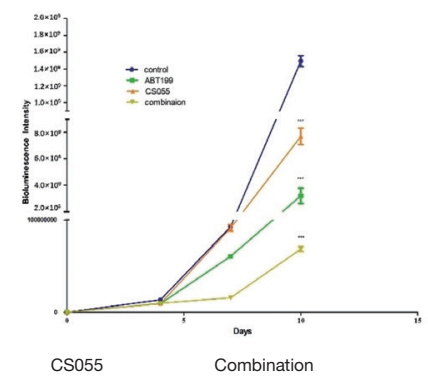

\section{C}
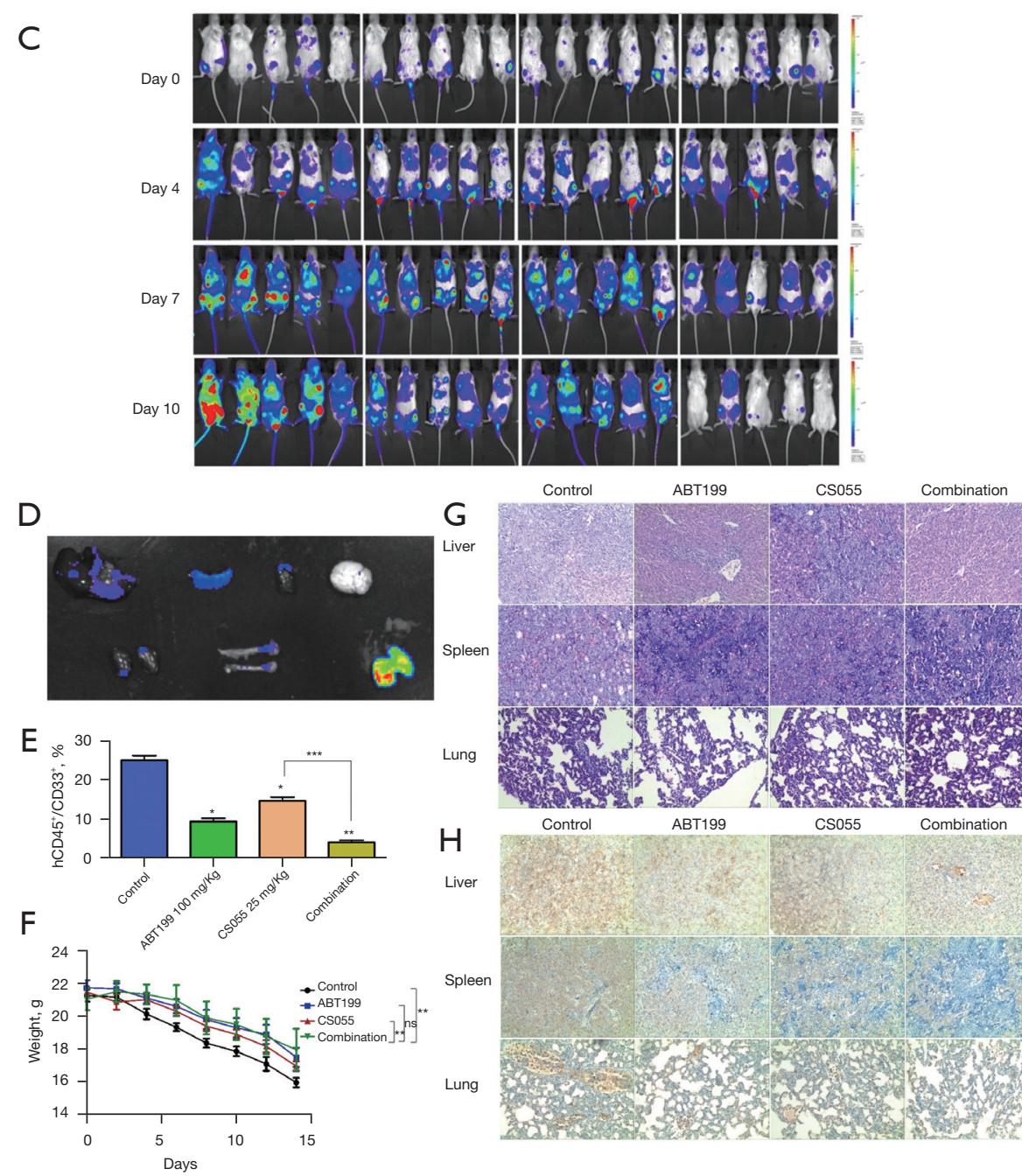

Combination

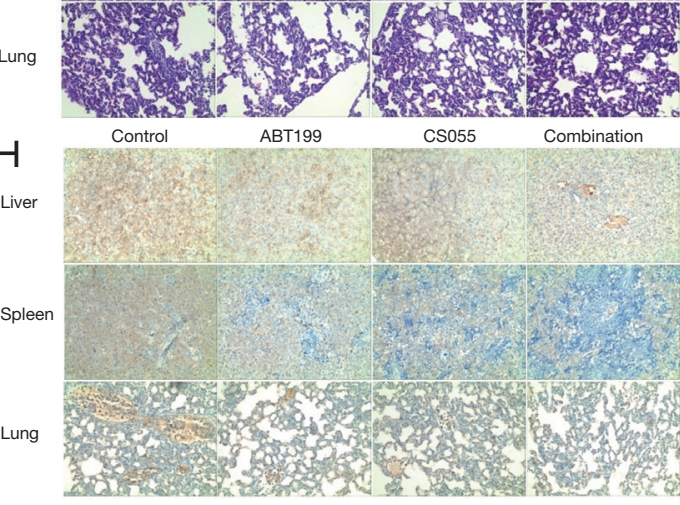

Figure 7 The effects of ABT199 (venetoclax) and CS055 (chidamide) on AML xenotransplantation mice, and the negative group was used as the control. (A) Survival curve of AML xenograft mice treated with ABT199 and CS055 alone or in combination. (B) Fluorescence intensity of AML xenograft mice in the monotherapy groups and the combination therapy group. (C) Tumorigenesis of AML xenograft mice treated with ABT199 and CS055 alone or in combination. (D) On the 10th day of administration, three mice in each group were sacrificed for in vivo imaging. (E) The number of $\mathrm{CD} 45^{+} \mathrm{CD} 33^{+}$cells in the bone marrow of AML xenotransplantation mice treated with $\mathrm{ABT} 199$ and CS055 alone or in combination for 10 days. (F) Body weight curve of mice with AML xenotransplantation treated with ABT199 and CS055 for 14 days. (G) Ten days later, mice were sacrificed, and the liver, spleen, and lung were stained with HE (magnification, $\times 200$ for liver and lung, $\times 400$ for spleen). (H) On the 10th day, the mice were sacrificed, and the liver, spleen and lung were stained for hCD45 ${ }^{+}$with immunohistochemistry (magnification: $\times 200$ for liver and lung, $\times 400$ for spleen). A $t$-test was used to analyze the data. *, $\mathrm{P}<0.05 ;{ }^{* *}, \mathrm{P}<0.01$; *** $\mathrm{P}<0.001$. ns, not significant; AML, acute myeloid leukemia. 
in the mitochondrial transmembrane potential and the release of JC-1 from the mitochondria. Early apoptosis can be detected by measuring the fluorescence intensity of JC-1 before morphological changes occur in apoptotic cells. Therefore, we detected changes in the mitochondrial membrane potential before and after treatment and found both venetoclax and chidamide decreased it in AML cells, and the effect was further enhanced after the two drugs were combined. This indirectly confirmed the synergistic effect of venetoclax and chidamide on apoptosis in AML cells.

The qRT-PCR results showed that Bcl-2, Mcl-1, and Bcl-xl also decreased after the two drugs were combined. These three genes are related to inhibiting apoptosis. Treatment with venetoclax resulted in an increase in Mcl1 protein levels in intrinsic drug-resistant AML cell lines, possibly because the binding of Mcl-1 and Bim increases the stability of Mcl-1 protein. The sequestration of Bim by Mcl-1 is a mechanism that drives the drug resistance to venetoclax in AML cells, and at the same time, Mcl1 may reduce the level of free Bim and prevent it from inducing apoptosis (34). Choudhary et al. (35) confirmed that an increased mRNA level and increased Mcl-1 protein stability led to increased protein levels in an acquired drugresistant AML cell line model. In our study, venetoclax monotherapy upregulated Mcl-1, while the expression of Mcl-1 decreased after combination therapy, which may be one of the mechanisms by which chidamide reduces resistance to venetoclax. While venetoclax reduced the association between Bcl-2 and Bim, once released, Bim/Mcl1 binding exhibited a compensatory increase, especially in venetoclax-resistant cell lines, preventing apoptosis (34). By reducing Bim/Mcl-1 and releasing Bim, Mcl-1 inhibition can terminate this association and eliminate venetoclax resistance (36-38), suggesting the combined inhibition of Mcl-1 and Bcl-2 is necessary to effectively induce apoptosis through this mechanism. Venetoclax decreased the binding of Bcl-2 to Bim but increased the binding of Mcl-1 to Bim, and Bim interacts with Bax/Bak, leading to Bax/Bak activation. Activated Bax/Bak forms pores in the outer membrane of mitochondria, leading to the release of cytochrome $\mathrm{c}$ and subsequent apoptosis. Bcl-2 was used to sequester Bim and prevent Bim from inducing apoptosis. The release of Bim from Bcl-2 by venetoclax reduced the association between $\mathrm{Bim}$ and $\mathrm{Bcl}-2$, which was offset by the increased interaction between Bim and Mcl1 , stabilizing Mcl-1 and leading to resistance to venetoclax. In venetoclax-resistant cells, treatment with venetoclax does not change the permeability of the mitochondrial outer membrane, which is a necessary event for intrinsic apoptosis. Mcl-1 has previously been shown to reduce DNA damage and to be necessary to inhibit Bak and Bax activation (39-42). Therefore, the change in the balance of Bcl-2 family members is the main cause of drug resistance. In our study, the downregulation of Bim was induced in both the chidamide monotherapy and combination therapy groups. Therefore, the binding of Bim to Mcl-1 decreased, which reduced the resistance to venetoclax. Schwartz et al. (32) confirmed that panobinostat upregulated Bim (a kind of HDACI), and in the presence of ABT-199, Bim still increased. We speculated there might be other mechanisms that drive Bim upregulation, which eventually led to an increase in Bax/Bak and apoptosis.

To determine how the expression of Mcl-1 protein was reduced by chidamide, we first studied it with qRT-PCR. At $24 \mathrm{~h}$ after treatment, the transcription level of Mcl-1 was significantly decreased by chidamide, and the decreased level was maintained in cells treated with the combination therapy (Figure 2D), indicating the transcription level of Mcl-1 was downregulated by chidamide. To determine whether chidamide affects the stability of Mcl-1 protein, we used chidamide and venetoclax alone or in combination to treat AML cell lines and primary cells for $24 \mathrm{~h}$. The results showed that the protein level of Mcl-1 was decreased in the chidamide monotherapy group and the combination therapy group, and the decrease was more obvious in the combination therapy group (Figure $2 F$ ). These results indicate that chidamide decreases the expression of Mcl-1 at both the transcriptional and protein levels. Venetoclax releases Bim from Bcl-2, but Bim can later be sequestered by Mcl-1. Therefore, the combined effect of Bcl-2 inhibition and Mcl-1 downregulation/inhibition is necessary to effectively induce apoptosis (34,36-38). Mcl1 is downregulated by chidamide, although the exact molecular mechanism remains undetermined, and we speculate that in venetoclax-sensitive cells, there may not be enough Mcl-1 to sequester all the released Bim, leading to the accumulation of free Bim and allowing it to activate $\mathrm{Bax} / \mathrm{Bak}$, leading to apoptosis. In contrast, in venetoclaxresistant cells, Bim released from Bcl-2 was sequestered by Mcl-1, which stabilized Mcl-1 and eventually led to the survival of venetoclax-resistant cells. Chidamide reduced the interaction between Mcl-1 and Bim, reduced the sequestration of Bim, allowed the activation of Bax/Bak, and eventually led to apoptosis.

It has been proven that JAK2/STAT3 signaling inactivation can inhibit growth and induce apoptosis in 
many cancer cells and tumors $(27,43,44)$. Therefore, the JAK2/STAT3 pathway is considered to be a target for therapy for many human cancers $(27,45,46)$. The JAKSTAT signaling pathway is widely activated in leukemia cells, with STAT1, STAT3, and STAT5 the most common signaling proteins. Myeloproliferative neoplasm (MPN) is characterized by the somatic acquisition of a mutation in either $7 A K 2$ ( $7 A K 2 \mathrm{~V} 617 \mathrm{~F})(47)$ or calreticulin (CALR) in a hematopoietic stem cell. SOCS3 protein, a negative regulator of JAK2/STAT3 signal transduction, plays a role as a tumor suppressor (48). We found that JAK2STAT3 signaling pathway-related genes were differentially expressed based on transcriptome sequencing, then verified that SOCS3 expression was upregulated, and $\mathcal{F} A K 2$ and STAT3 gene expression was downregulated with qRTPCR. At the same time, we further verified the correlation at the protein level with Western blot analysis, which also showed the expression of SOCS3 was upregulated, and the expression of P-JAK2 and P-STAT3 was downregulated. Therefore, we concluded that the JAK2/STAT3 signaling pathway was inhibited. The downstream related factors in the STAT3 pathway can regulate the cell cycle and apoptosis process, and inhibit the expression of the CDK2 and c-myc genes which are downstream of STAT3, by upregulating the expression of $\mathrm{p} 21$. At the same time, it inhibited the expression of Bcl-2 and Mcl-1 and promoted apoptosis.

Mcl-1 are also downstream of the PI3K/AKT pathway, which are frequently constitutively activated in AML $(8,49)$. The PI3K/AKT signaling pathway plays a key role in tumor occurrence, development, and radiation resistance, is involved in various cellular activities and metabolic regulation, and is closely related to the proliferation and apoptosis, cell cycle regulation, angiogenesis, invasion, and metastasis of cancer cells. The PI3K/AKT pathway inhibits apoptosis through a variety of mechanisms (50), and AKT upregulates the expression of c-myc by upregulating the transcription of c-myc. AKT can also inhibit GSK3 $\beta$ kinase activity via phosphorylation to prevent cyclin D1 degradation, change the cell cycle distribution, and promote cell proliferation in S phase. We found differences in the expression of PI3K-AKT pathway-related genes after chidamide was combined with venetoclax. By qRT-PCR, we verified that AKT gene expression was downregulated in this pathway, while Western blot analysis was used to verify that chidamide reduced the protein expression of P-AKT. After combination treatment with venetoclax, the P-AKT level further decreased, and the activation of the PI3K-AKT pathway was inhibited. Through the upregulation of $\mathrm{p} 21$ protein expression, the expression of CDK2 and myc was downregulated, which caused cell cycle arrest. These results show chidamide can inhibit the activation of the PI3K-AKT signaling pathway to have anti-AML effects by cooperating with venetoclax.

Regarding cell cycle regulation, the $p 21$ gene controls the synthesis of $\mathrm{p} 21$ protein in human cells, which can inhibit DNA replication. Venetoclax and chidamide can increase the $p 21$ gene and protein levels, and chidamide can also inhibit the PI3K/AKT pathway. Western blot results showed that $\mathrm{P}-\mathrm{AKT}$ protein was downregulated after the addition of chidamide, and CDK2 was an important regulator of the G1/S phase transition. On the other hand, the downregulation of P-AKT also led to the downregulation of c-myc, which is a gene that promotes cell division, and c-myc is also a downstream factor of STAT3 in the JAK2/STAT3 pathway. After the JAK2/STAT3 pathway is inhibited, CDK2 and c-myc are downregulated, which together cause cell cycle arrest and inhibit the proliferation of AML cells.

Moreover, the expression of HDAC1 was upregulated, resulting in the prevention of histone deacetylation. Thus, the degree of histone acetylation increased, and cell proliferation was inhibited by upregulating $\mathrm{p} 21$ protein levels.

\section{Conclusions}

Our study generated three conclusions. First, venetoclax combined with chidamide can synergistically promote apoptosis in AML cell lines and primary cells. Second, transcriptome sequencing, qRT-PCR, and Western blot shows that chidamide synergistically promotes the apoptosis of AML cells by inhibiting the activation of the PI3K/AKT pathway and JAK2/STAT3 pathway. Third, compared with the monotherapy group, the combination of chidamide and venetoclax significantly inhibits tumor progression and prolonged the survival time of mice. The purpose of this study was to explore the antileukemia effect and mechanism of action of venetoclax combined with chidamide. The research results of this study provide a new strategy and theoretical basis for solving the problem of drug resistance to venetoclax in clinical practice.

\section{Acknowledgments}

The work was supported by the Department of 
Hematopathy, Henan Institute of Hematology, The Affiliated Cancer Hospital of Zhengzhou University and we would like to sincerely thank all staff involved.

Funding: This study was supported by the Henan Province Science and Technology Research Project (No. 202102310053$)$, Henan Province Science and Technology Research Project (No. LHGJ20200168), Henan Province Science and Technology Research Project (No. 192102310056), and Henan Province Science and Technology Research Project (No. 192102310053).

\section{Footnote}

Reporting Checklist: The authors have completed the ARRIVE reporting checklist. Available at https://dx.doi. org/10.21037/atm-21-5066

Data Sharing Statement: Available at https://dx.doi. org/10.21037/atm-21-5066

Conflicts of Interest: All authors have completed the ICMJE uniform disclosure form (available at https://dx.doi. org/10.21037/atm-21-5066). The authors have no conflicts of interest to declare.

Ethical Statement: The authors are accountable for all aspects of the work in ensuring that questions related to the accuracy or integrity of any part of the work are appropriately investigated and resolved. All procedures performed in this study involving human participants were in accordance with the Declaration of Helsinki (as revised in 2013). The study was approved by the Medical Ethics Committee of The Affiliated Cancer Hospital of Zhengzhou University (approval No. 2021-KY-0170-001) and informed consent was taken from all the patients. Experiments were performed under a project license (approval No. 20200320) granted by Ethics Review Committee of life sciences, Zhengzhou University, in compliance with Zhengzhou University national or institutional guidelines for the care and use of animals.

Open Access Statement: This is an Open Access article distributed in accordance with the Creative Commons Attribution-NonCommercial-NoDerivs 4.0 International License (CC BY-NC-ND 4.0), which permits the noncommercial replication and distribution of the article with the strict proviso that no changes or edits are made and the original work is properly cited (including links to both the formal publication through the relevant DOI and the license). See: https://creativecommons.org/licenses/by-nc-nd/4.0/.

\section{References}

1. Dombret H, Gardin C. An update of current treatments for adult acute myeloid leukemia. Blood 2016;127:53-61.

2. Tamamyan G, Kadia T, Ravandi F, et al. Frontline treatment of acute myeloid leukemia in adults. Crit Rev Oncol Hematol 2017;110:20-34.

3. Bose P, Vachhani P, Cortes JE. Treatment of Relapsed/ Refractory Acute Myeloid Leukemia. Curr Treat Options Oncol 2017;18:17.

4. Konopleva M, Pollyea DA, Potluri J, et al. Efficacy and Biological Correlates of Response in a Phase II Study of Venetoclax Monotherapy in Patients with Acute Myelogenous Leukemia. Cancer Discov 2016;6:1106-17.

5. DiNardo CD, Rausch CR, Benton C, et al. Clinical experience with the BCL2-inhibitor venetoclax in combination therapy for relapsed and refractory acute myeloid leukemia and related myeloid malignancies. Am J Hematol 2018;93:401-7.

6. Yogarajah M, Stone RM. A concise review of BCL-2 inhibition in acute myeloid leukemia. Expert Rev Hematol 2018;11:145-54.

7. Pan R, Hogdal LJ, Benito JM, et al. Selective BCL-2 inhibition by ABT-199 causes on-target cell death in acute myeloid leukemia. Cancer Discov 2014;4:362-75.

8. Wang $\mathrm{X}, \mathrm{Mak} \mathrm{PY}, \mathrm{Mu} \mathrm{H}$, et al. Combinatorial Inhibition of Focal Adhesion Kinase and BCL-2 Enhances Antileukemia Activity of Venetoclax in Acute Myeloid Leukemia. Mol Cancer Ther 2020;19:1636-48.

9. Ruefli-Brasse A, Reed JC. Therapeutics targeting Bcl-2 in hematological malignancies. Biochem J 2017;474:3643-57.

10. Yalniz FF, Wierda WG. Targeting BCL2 in Chronic Lymphocytic Leukemia and Other Hematologic Malignancies. Drugs 2019;79:1287-304.

11. DiNardo CD, Pratz K, Pullarkat V, et al. Venetoclax combined with decitabine or azacitidine in treatmentnaive, elderly patients with acute myeloid leukemia. Blood 2019;133:7-17.

12. DiNardo CD, Pratz KW, Letai A, et al. Safety and preliminary efficacy of venetoclax with decitabine or azacitidine in elderly patients with previously untreated acute myeloid leukaemia: a non-randomised, open-label, phase 1b study. Lancet Oncol 2018;19:216-28.

13. Xu L, Xu Y, Jing Z, et al. Altered expression pattern of miR-29a, miR-29b and the target genes in myeloid 
leukemia. Exp Hematol Oncol 2014;3:17.

14. Vela L, Gonzalo O, Naval J, et al. Direct interaction of Bax and Bak proteins with Bcl-2 homology domain 3 (BH3)-only proteins in living cells revealed by fluorescence complementation. J Biol Chem 2013;288:4935-46.

15. Wang M, Wu D, Liu P, et al. Silence of MCL-1 upstream signaling by shRNA abrogates multiple myeloma growth. Exp Hematol Oncol 2014;3:27.

16. Cho H, Jang JE, Eom JI, et al. Arsenic trioxide synergistically promotes the antileukaemic activity of venetoclax by downregulating Mcl-1 in acute myeloid leukaemia cells. Exp Hematol Oncol 2021;10:28.

17. Mistry JJ, Hellmich C, Lambert A, et al. Venetoclax and Daratumumab combination treatment demonstrates pre-clinical efficacy in mouse models of Acute Myeloid Leukemia. Biomark Res 2021;9:35.

18. Ramsey HE, Fischer MA, Lee T, et al. A Novel MCL1 Inhibitor Combined with Venetoclax Rescues VenetoclaxResistant Acute Myelogenous Leukemia. Cancer Discov 2018;8:1566-81.

19. Falkenberg KJ, Johnstone RW. Histone deacetylases and their inhibitors in cancer, neurological diseases and immune disorders. Nat Rev Drug Discov 2014;13:673-91.

20. Roy DM, Walsh LA, Chan TA. Driver mutations of cancer epigenomes. Protein Cell 2014;5:265-96.

21. Brodska B, Holoubek A, Otevrelova P, et al. Combined treatment with low concentrations of decitabine and SAHA causes cell death in leukemic cell lines but not in normal peripheral blood lymphocytes. Biomed Res Int 2013;2013:659254.

22. Tan P, Wei A, Mithraprabhu S, et al. Dual epigenetic targeting with panobinostat and azacitidine in acute myeloid leukemia and high-risk myelodysplastic syndrome. Blood Cancer J 2014;4:e170.

23. Liu L, Chen B, Qin S, et al. A novel histone deacetylase inhibitor Chidamide induces apoptosis of human colon cancer cells. Biochem Biophys Res Commun 2010;392:190-5.

24. Shi Y, Dong M, Hong X, et al. Results from a multicenter, open-label, pivotal phase II study of chidamide in relapsed or refractory peripheral T-cell lymphoma. Ann Oncol 2015;26:1766-71.

25. Xie A, Liao C, Li Z, et al. Quantitative structure-activity relationship study of histone deacetylase inhibitors. Curr Med Chem Anticancer Agents 2004;4:273-99.

26. Shi Y, Jia B, Xu W, et al. Chidamide in relapsed or refractory peripheral $\mathrm{T}$ cell lymphoma: a multicenter realworld study in China. J Hematol Oncol 2017;10:69.
27. Zhao S, Guo J, Zhao Y, et al. Chidamide, a novel histone deacetylase inhibitor, inhibits the viability of MDS and AML cells by suppressing JAK2/STAT3 signaling. Am J Transl Res 2016;8:3169-78.

28. Wang H, Liu YC, Zhu CY, et al. Chidamide increases the sensitivity of refractory or relapsed acute myeloid leukemia cells to anthracyclines via regulation of the HDAC3 -AKT-P21-CDK2 signaling pathway. J Exp Clin Cancer Res 2020;39:278.

29. Huang H, Wenbing Y, Dong A, et al. Chidamide Enhances the Cytotoxicity of Cytarabine and Sorafenib in Acute Myeloid Leukemia Cells by Modulating H3K9me3 and Autophagy Levels. Front Oncol 2019;9:1276.

30. Pan R, Ruvolo V, Mu H, et al. Synthetic Lethality of Combined Bcl-2 Inhibition and p53 Activation in AML: Mechanisms and Superior Antileukemic Efficacy. Cancer Cell 2017;32:748-60 e6.

31. Gong K, Xie J, Yi H, et al. CS055 (Chidamide/HBI-8000), a novel histone deacetylase inhibitor, induces G1 arrest, ROS-dependent apoptosis and differentiation in human leukaemia cells. Biochem J 2012;443:735-46.

32. Schwartz J, Niu X, Walton E, et al. Synergistic antileukemic interactions between ABT-199 and panobinostat in acute myeloid leukemia ex vivo. Am J Transl Res 2016;8:3893-902.

33. Kroemer G, Zamzami N, Susin SA. Mitochondrial control of apoptosis. Immunol Today 1997;18:44-51.

34. Niu X, Zhao J, Ma J, et al. Binding of Released Bim to Mcl-1 is a Mechanism of Intrinsic Resistance to ABT199 which can be Overcome by Combination with Daunorubicin or Cytarabine in AML Cells. Clin Cancer Res 2016;22:4440-51.

35. Choudhary GS, Al-Harbi S, Mazumder S, et al. MCL-1 and BCL-xL-dependent resistance to the BCL-2 inhibitor ABT-199 can be overcome by preventing PI3K/AKT/ mTOR activation in lymphoid malignancies. Cell Death Dis 2015;6:e1593.

36. Luedtke DA, Niu X, Pan Y, et al. Inhibition of Mcl1 enhances cell death induced by the Bcl-2-selective inhibitor ABT-199 in acute myeloid leukemia cells. Signal Transduct Target Ther 2017;2:17012.

37. Moujalled DM, Pomilio G, Ghiurau C, et al. Combining BH3-mimetics to target both BCL-2 and MCL1 has potent activity in pre-clinical models of acute myeloid leukemia. Leukemia 2019;33:905-17.

38. Teh TC, Nguyen NY, Moujalled DM, et al. Enhancing venetoclax activity in acute myeloid leukemia by cotargeting MCL1. Leukemia 2018;32:303-12. 
39. Jamil S, Mojtabavi S, Hojabrpour P, et al. An essential role for MCL-1 in ATR-mediated CHK1 phosphorylation. Mol Biol Cell 2008;19:3212-20.

40. Bose P, Grant S. Mcl-1 as a Therapeutic Target in Acute Myelogenous Leukemia (AML). Leuk Res Rep 2013;2:12-4.

41. Nijhawan D, Fang M, Traer E, et al. Elimination of Mcl1 is required for the initiation of apoptosis following ultraviolet irradiation. Genes Dev 2003;17:1475-86.

42. Cuconati A, Mukherjee C, Perez D, et al. DNA damage response and MCL-1 destruction initiate apoptosis in adenovirus-infected cells. Genes Dev 2003;17:2922-32.

43. Corcoran RB, Contino G, Deshpande V, et al. STAT3 plays a critical role in KRAS-induced pancreatic tumorigenesis. Cancer Res 2011;71:5020-9.

44. Yao H, Ma Y, Hong Z, et al. Activating JAK2 mutants reveal cytokine receptor coupling differences that impact outcomes in myeloproliferative neoplasm. Leukemia 2017;31:2122-31.

45. Zehender A, Huang J, Gyorfi AH, et al. The tyrosine phosphatase SHP2 controls TGFbeta-induced STAT3 signaling to regulate fibroblast activation and fibrosis. Nat

Cite this article as: Li G, Li D, Yuan F, Cheng C, Chen L, Wei X. Synergistic effect of chidamide and venetoclax on apoptosis in acute myeloid leukemia cells and its mechanism. Ann Transl Med 2021;9(20):1575. doi: 10.21037/atm-21-5066
Commun 2018;9:3259.

46. Xie YL, Tao WH, Yang TX, et al. Anticancer effect of cucurbitacin B on MKN-45 cells via inhibition of the JAK2/STAT3 signaling pathway. Exp Ther Med 2016;12:2709-15.

47. Baxter EJ, Scott LM, Campbell PJ, et al. Acquired mutation of the tyrosine kinase JAK2 in human myeloproliferative disorders. Lancet 2005;365:1054-61.

48. He B, You L, Uematsu K, et al. SOCS-3 is frequently silenced by hypermethylation and suppresses cell growth in human lung cancer. Proc Natl Acad Sci U S A 2003;100:14133-8.

49. Yamaguchi H, Hsu JL, Hung MC. Regulation of ubiquitination-mediated protein degradation by survival kinases in cancer. Front Oncol 2012;2:15.

50. Kumar D, Haldar S, Gorain M, et al. Epoxyazadiradione suppresses breast tumor growth through mitochondrial depolarization and caspase-dependent apoptosis by targeting PI3K/Akt pathway. BMC Cancer 2018;18:52.

(English Language Editor: B. Draper) 